Ann. Génét. Sél. anim., I978, 10 (I), I3I-I34.

Note

\title{
Anomalies congénitales héréditaires ou non en race bovine Normande
}

\author{
J. J. LAUUVERGNE \\ Département de Génétique animale, \\ Centre national de Recherches zootechniques, I.N.R.A. \\ $7835^{\circ}$ Jouy-en-Josas
}

\section{Résumé}

On a tout d'abord décelé en race Normande une forme héréditaire de polydactylie. Plus récemment le déterminisme exact d'une hypotrichose assez fréquente n'a pas encore été établie avec certitude. En outre un syndrome d'anourie/anophthalmie n'est vraisemblablement pas héréditaire. Les procédés d'enquête utilisés sont encore très légers et ne décèlent qu'une faible partie des cas. On préconise, comme en Charolais, un examen systématique des veaux nés au cours des opérations de testage sur la descendance.

En France depuis près de vingt ans le Département de Génétique animale de I'I.N.R.A., en liaison avec les spécialistes des Écoles Vétérinaires, les organismes

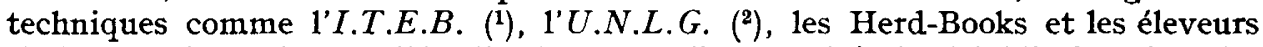
s'efforcent de repérer et d'étudier les anomalies congénitales héréditaires dans les différentes races bovines.

Dans la race Normande on s'est tout d'abord intéressé, au début des années soixante, à des cas de polydactylie apparus dans la descendance d'un taureau en testage, à l'époque, au centre de Charmoy (Yonne). L'animal était parfaitement normal et les femelles auxquelles il avait été accouplées elles aussi normales et choisies au hasard. Il y avait des témoins (tous les descendants des autres taureaux mis au testage en même temps) dont aucun n'était polydactyle. La présomption

(I) I.T.E.B. Institut technique de 1'Élevage bovin, Paris.

(2) U.N.I.G. Union nationale des Livres généalogiques, Paris. 
en faveur d'une étiologie héréditaire génique était donc forte, mais il était difficile de trancher entre la monofactorialité récessive ou la dominance à pénétrance incomplète (LAUVERGNE, I962). Par la suite, ce défaut n'a pas été de nouveau signalé, ce qui n'a pas permis d'en préciser le déterminisme.

Après cette alerte, le centre de Charmoy a mis en place un système de repérage léger des anormaux dans sa zone, suivi en cela par d'autres coopératives ( $L_{\text {'Aigle, }}$ Mayenne, Magny-en-Vexin) cependant que des renseignements nous parvenaient par diverses autres sources : lettres d'éleveurs adressées au Centre National de Recherches Zootechniques, articles dans des journaux locaux signalés par divers correspondants, etc.

C'est ainsi que notre attention a tout d'abord été attirée par une série d'anomalies plus ou moins associées : anophthalmie (absence d'yeux), anourie (absence de queue), anomalie des pattes, absence d'anus, puis, à partir de 1967 , par 1'hypotrichose (absence presque totale de poils). Cinq cas de cette dernière anomalie étaient déjà connus au début de I97 I lorsqu'il fut décidé d'entreprendre une étude plus systématique avec enquête dans la zone Normande et élevage de proposants. On pouvait en effet penser que certaines au moins de ces anomalies étaient héréditaires monofactorielles, surtout 1'hypotrichose.

Un premier veau sans poils, une femelle, fut achetée puis, en I972, une collaboration s'établit avec le Service de Zootechnie de 1'École Vétérinaire d'Alfort pour l'élevage et les croisements expérimentaux. Au début de I973 une enquête "anomalies en race Normande " était lancée par voie de presse. Trois articles dans Paris Normandie et quatre articles dans Owest-France entre février 1973 et janvier I974 suscitaient au total 25 réponses intéressant la Normande, dont 8 cas d'hypotrichoses, 5 cas d'anophthalmie, 4 cas d'anourie et 4 cas d'anourie associée à l'anophthalmie. Cela confirmait le " profil anomalique " de la Normande avec une dominance de 1'anomalie associée (anophthalmie/anourie).

En ce qui concerne 1'hypotrichose, à partir des animaux rassemblés à Alfort, on a pu étudier les caractéristiques anatomiques et histologiques de l'anomalie et procéder à des croisements. Un premier article descriptif est paru (DENIs et al.; I975) montrant que tous les anomaliques présentaient bien le même type d'hypotrichose, ce qui est un argument au moins en faveur d'une commune étiologie. Cependant les accouplements entre hypotrichosiques n'ont pas donné d'anormatix; ce qui laisse à penser que, si l'anomalie est héréditaire, elle ne peut être que récessive à pénétrance incomplète. Par ailleurs les pères des hypotrichosiques ne présentent pas de relation de parenté proches.

Les cas décelés d'anourie et/ou de microphthalmie/anophthlamie sont présentés dans le tableau I. On a mentionné les principaux défauts associés ainsi que les cas observés dans d'autres races.

L'analyse des demi-frères des anoures anophthalmiques en Normand n'a pas révélé d'accumulation particulière dans des descendances, sauf un cas mais le taureau avait été très utilisé. L'anourie a été souvent étudiée individuellement mais RIECK (I966) a montré qu'elle n'était simple que dans 27 p. Ioo des cas et qu'il y: avait lieu de distinguer 4 syndromes : r) brachyurie, anourie; 2) syndrome:caudo-recto urogénita1;3) syndrome d'anourie et d'anophthalmie; 4) anourie ou brachyourie et autres défauts. Ces différents syndromes se retrouvent dans notre échantillon. De même, après Huston et WEARDEN (I958) on note que les anomalies caudales sont au moins deux fois plus fréquentes chez les femelles que chez les mâles. Étudiant quant à eux l'anophthalmie et la microphthalmie LEIPOLD et Huston (I968) ont trouvé ro cas sur 26 avec des anomalies caudales, ce qui correspond, là aussi, assez bien avec les fréquences que nous observons. 
Incontestablement on constate une association entre les localisations oculaires et caudales, ce qui témoigne sans doute d'une étiologie commune mais, jusqu'à présent les auteurs étrangers qui ont analysé génétiquement les données et même dans certains cas fait des expériences de croisement (GILMORE et FEChHEIMER, I957; Huston et WEARDEN, I958; LEIPOLD et Huston, I968) n'ont pu déceler une influence héréditaire, sinon une légère prédisposition dans certaines races. La présence de cette anomalie dans une race non encore étudiée sous cet angle, la Normande, vient encore à l'appui d'une étiologie non mendélienne.

Parmi les autres cas d'anomalies signalées en race Normande et qui pourraient être héréditaires, il y a un veau albinos, né en 1977.

\section{TABLEAU I}

Anourie et anophthalmie dans la race Normande $c t$ dans quelques autres vaces françaises

Anouria and Anophthalmia in Norman cattle and in some other French breeds

\begin{tabular}{|c|c|c|c|c|c|c|c|c|c|c|c|}
\hline \multirow[t]{2}{*}{ Race } & \multicolumn{4}{|c|}{ Anourie } & \multicolumn{3}{|c|}{$\begin{array}{l}\text { Anophthalmie ou } \\
\text { microphthalmie }\end{array}$} & \multicolumn{4}{|c|}{$\begin{array}{c}\text { Anourie et } \\
\text { anophthalmie }\end{array}$} \\
\hline & MI & $\mathrm{F}$ & $\begin{array}{l}\text { (1) } \\
i_{i}\end{array}$ & $\begin{array}{l}\text { Sexe } \\
\text { non. } \\
\text { préc. }\end{array}$ & $\mathbf{M}$ & $F$ & $\begin{array}{l}\text { Sexe } \\
\text { non } \\
\text { préc. }\end{array}$ & $M$ & $\mathrm{~F}$ & (') & $\begin{array}{l}\text { Sexe } \\
\text { non } \\
\text { préc. }\end{array}$ \\
\hline $\begin{array}{l}\text { Normande. } \\
\text { Frisonne . } \\
\text { Charolaise } \\
\text { Maine-Anjou } \\
\text { Croisés . }\end{array}$ & $\begin{array}{l}3 \\
1\end{array}$ & $\begin{array}{l}1 \text { I }\left({ }^{2}\right) \\
5 \\
2 \\
2 \\
4\end{array}$ & $\cdots$ & 4 & 4 & $\begin{array}{r}10 \\
1\end{array}$ & I & $\begin{array}{l}5 \\
\text { I }\end{array}$ & $\begin{array}{l}4 \\
z \\
\mathrm{I}\end{array}$ & I & I \\
\hline & 7 & 24 & 2 & 5 & 5 & I I & I & 8 & 9 & I & I \\
\hline
\end{tabular}

(I) Il s'agit d'animaux avec des anomalies englobant la région génito-anale ce qui empêche de préciser le sexe.

(2) 2 des femelles avaient l'anus éctopique et une autre presentait seulement l'anomalie anale.

Finalement le moins qu'on puisse dire, c'est que les études sur l'étiologie des tares congénitales en race Normande demandent encore bien des précisions. On pourrait dans cette race appliquer la méthode de l'analyse des fratries de demigermains (les lots de descendants des taureaux de testage) présentée par LAUVERGNE et LEFORT (I973) et utilisé à résoudre le problème de SAP en Charolais, LAUVERGNE (I974), LEFORT et al. (I977). Avec les I50 taurillons mis en testage annuellement on aurait rapidement assez d'information. Malheureusement le testage sur la descendance en Normand est un testage lait qui n'est pas conçu pour l'examen systématique des veaux à la naissance, comme 1'est le testage viande du Charolais. 


\title{
Summary
}

\author{
Hereditary and non hereditary congenital abnormalities in Normandy cattle
}

Congenital polydactyly was the first hereditary defect detected in the Normandy breed. A type of hypotrichosis has then been observed but the degree and mode of its inheritance have not been established. A syndrome with anouria and/or anophthalmia is probably non hereditary. The survey in its present form is able to detect only a few cases. A systematic checking of all calves born in progeny testing programs is proposed to increase the data base for more critical analysis.

\section{Références bibliographiques}

Denis B., Thíke't M., Blin P. C., Bernard C., Lauvergne J. J., 1975. Hypotrichose congénitale en race Normande. 1. Étude descriptive. Ann. Génét. Sél. anim., 7, 25I-26I.

GILMORE L. O., Fechreimer N. S., r957. Tailless calves studied in attempt to determine cause of abnormality. Ohio Farm and Home Research, 42, 32-33.

HUSTON K., WeARden S., I958. Congenital taillessness in cattle. J. Dairy Sci., 41, r359-1370.

LAUvergne J. J., i962. Nouveau cas de polydactylie héréditaire chez les Bovins. Ann. Zootech., 11, I 5 I-I 56.

LAUVERGNE J. J., r974. Étude des gènes à effets visibles à partir des données de testage des taureaux sur leur descendance. ${ }^{\mathrm{er}}$ Congr. Mond. Génét. appl. Elevage, Madrid, 1, 47-55.

LAUVERGNe J. J., LEForT G, r973. Nouvelle méthode pour analyser le comportement et la fréquence des gènes récessifs à effets visibles dans les populations bovines. C. R.Acad.Sci.Paris, Sér. $D, 277$ (24), 2793-2796.

Lefort G., LaUvergne J. J., Fabrègue P., i977. Étude du gène responsable du Syndrome d'Arthrogrypose et de Palatoschisis dans la race Charolaise en France : fréquence génique et pénétrances. Ann. Génét. Sél. anim., 9, z83-30o.

LEIPOLD H. W., HUSTON K., 1968. Congenital Syndrome of Anophthalmia-Microphthalmia with associated defects in cattle. Path. Vet., 5, 407-418.

RIECK G. W., I966. Über Schwanzlosigkeit beim Rind. Dtsch. Tierärztl. Wochenschr., 73 (4), 8o-88. 